\title{
Animal-free alternatives and the antibody iceberg
}

To the Editor - Antibody generation using animal immunization presents scientific and ethical concerns. From a scientific standpoint, animal-generated antibodies can suffer from batch-to-batch variation, low specificity, high background and undefined identity of the binding reagent - all of which can compromise research reproducibility. From an ethical standpoint, the European Union (EU) core values are to replace, reduce or refine animal use in research and improve the quality of research. Advanced in vitro technologies for antibody generation not only offer greater versatility and reproducibility over animal immunization, but also alleviate ethical concerns. Yet for many researchers and antibody reagent manufacturers or suppliers, non-animal-based approaches remain underutilized - with a vast portion of the entirety of molecules in the antibody iceberg' remaining under their sonar (Fig. 1).

Motivated by the scientific and ethical issues posed by animal-derived antibodies ${ }^{1,2}$ - and European Union (EU) Directive $2010 / 63 / \mathrm{EU}^{3}$, which requires the use of animal-free replacement methods if available and scientifically justified - the EU Reference Laboratory for Alternatives to Animal Testing (EURL ECVAM) convened a scientific advisory committee (ESAC) working group to "review the scientific validity of non-animal-derived antibodies and non-antibody affinity reagents used for research, regulatory applications and diagnostics." At the request of ECVAM, the ESAC excluded from its analysis any antibodies to be administered to human patients. The ESAC notes that the generation of antibodies from universal libraries or from immunized animals (including transgenic animals), converted into recombinant format, are both well established for therapeutic uses. The report concluded "that non-animal derived antibodies are mature reagents generated by a proven technology that are not only equivalent to animal-derived antibodies, but in many respects can offer significant scientific advantages and economic benefits." The EURL ECVAM released its recommendation on non-animal-derived antibodies in $\mathrm{May}^{4}$. Collectively, the ESAC report and opinion, as well as the EURL ECVAM recommendation, form the European Commission's Joint Research Centre (JRC) science-for-policy report. Even though the ESAC review did not cover the field of therapeutic applications, the
EURL ECVAM recommendation considers non-animal-derived antibodies to be a suitable alternative in this field as well, in accordance with the directive.

Overall, the JRC report urges all scientific sectors, government authorities, funding agencies and publishers to endorse and adopt technologies for the generation of non-animal-derived antibodies. While the technologies are mature, widespread adoption remains logistically challenging at this time, so the goal to replace the use of animal-derived antibodies in research is an aspirational one, limited by present access to this technology, both in individual research laboratories and with commercial antibody providers. EURL ECVAM has not recommended that existing well-characterized hybridomas should be abandoned, as animals are no longer involved in their production. Preferably, they should be sequenced and used to produce recombinant monoclonal antibodies (mAbs) in vitro. As the exclusive use of recombinant antibodies will lead to better science, it should be promoted and implemented in any possible way that does not scientifically restrain research.

Here we outline the report's main findings and why it is expected to have major implications for how antibodies are generated for the basic research market and for biomedical and other industrial applications.

\section{Out of mind, out of sight}

Well-characterized antibodies have high specificity for their antigens and are essential tools used across all scientific disciplines. The research market is heavily dominated by animal-derived antibodies, yet is unable to supply high-quality reagents across the board, resulting in grave scientific concerns related to lack of reproducibility and specificity ${ }^{5-7}$. Fortunately, techniques that rely on animal immunization represent just the tip of the antibody iceberg: a vast expanse of additional molecules can be accessed by advanced recombinant methods that largely remain out of sight (Fig. 1).

Universal (non-animal-derived) antibody libraries contain an enormous repertoire of structurally diverse antibody genes equal to, or larger than, that of a naive immune system, from which antibodies against essentially any target can be generated. Recombinant antibodies produced from such libraries benefit from being unambiguously and routinely identified by their sequence. Knowledge of the sequence allows resynthesis of an antibody gene at any time, providing a consistently reliable supply - something that cannot be achieved by hybridoma technology, where loss of a producer cell line can be catastrophic. Furthermore, in vitro antibody selection against an antigen can be more tightly controlled than an immunization, to enrich clones with desired properties, such as specificity, stability, yield or affinity.

Given the availability of recombinant methods to generate antibodies from universal display libraries, such as phage display - a technology in use for 29 years and recognized with a Nobel Prize in 2018 - or yeast display, the reluctance to dispense with antibodies generated by obsolescent animal immunization technology, with its well-documented limitations, appeared to be something of a conundrum. Newer antibody generation technologies offer substantial time benefits, far greater versatility and substantially improved reproducibility. Yet, for most life science researchers, suppliers and manufacturers, these antibody library-based methods remain underused and beyond their vision - akin to the submerged portion of the antibody iceberg (Fig. 1). The key factor preventing their uptake is today's limited commercial availability.

This was the issue considered by the JRC EURL ECVAM ${ }^{1-3}$. To approach this complex problem from all relevant viewpoints, the ESAC brought together experienced scientists from the academic and commercial sectors, with expertise in diagnostics, therapeutics, research, antibody generation using animal and non-animal technologies, antibody engineering, and downstream immuno-analysis applications. (The authors of this Correspondence were all ad hoc members of the ESAC.) Based on the existing scientific literature, application examples and the ESAC's own extensive experience, the mission was to review the majority of uses where animal-derived antibodies are relied on and evaluate the benefits and limitations associated with their use, along with the benefits and limitations of their proposed non-animal-derived replacements.

The findings from ESAC's review of "the scientific validity of replacements for animal-derived antibodies" - which led to the EURL ECVAM "Recommendation on non-animal derived antibodies" ${ }^{4}$ - are disturbing. Animal-derived antibodies 


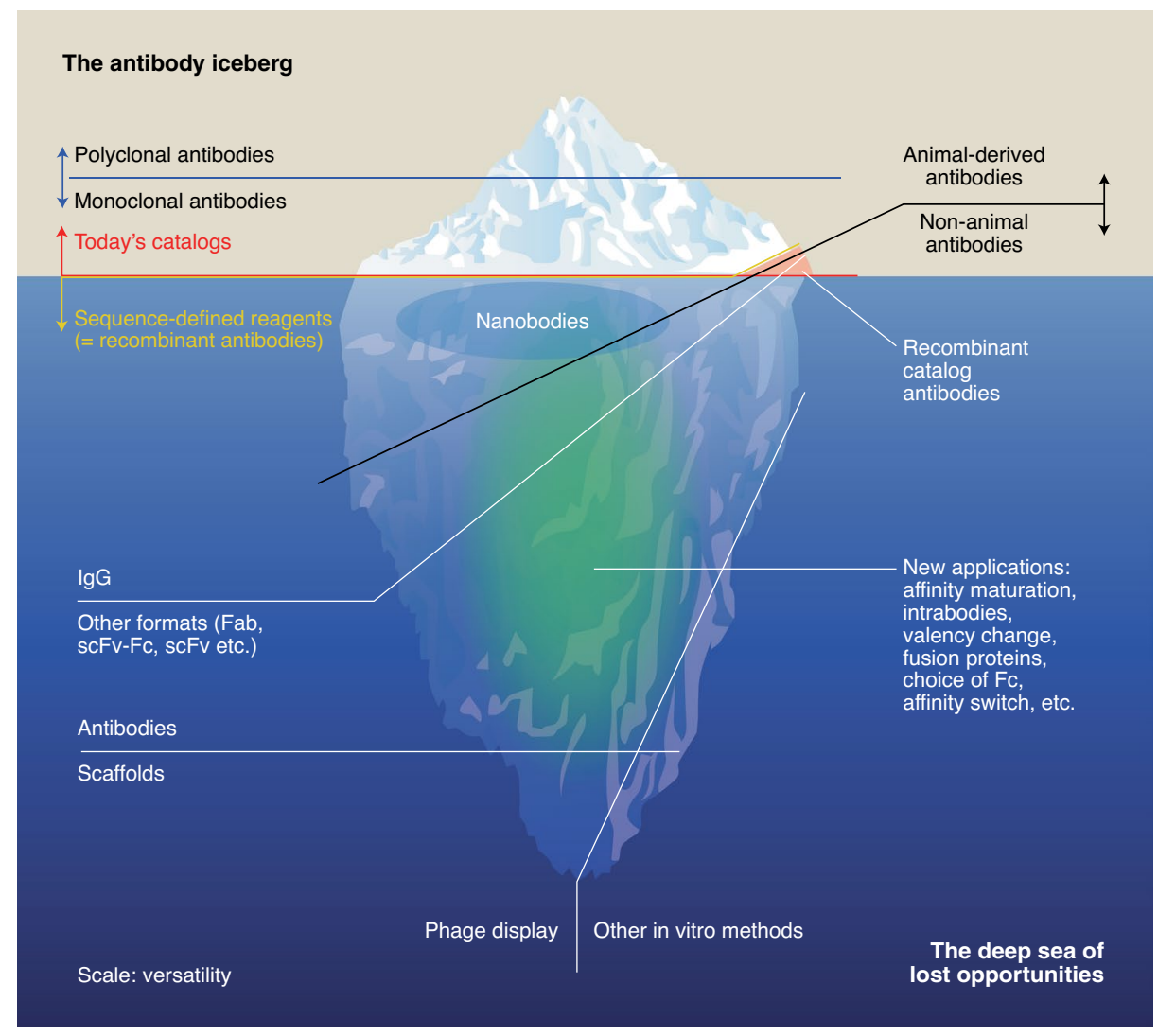

Fig. 1 | The antibody iceberg. Only a tiny tip of the potential of antibody generation methods are now exploited. This tip comprises visible, widely utilized animal immunization-based methods. The submerged fractions comprising in vitro recombinant antibody generation methods present a wealth of opportunities yet to be exploited to their full potential and lacking visibility outside the development of therapeutics. Colored lines divide the iceberg into the approximate fractions each antibody technology occupies. Antibodies derived from animal immunization (above black line) are divided into polyclonal, monoclonal and sequence-defined fractions. The polyclonal and monoclonal fractions (dark blue line) occupy the visible fraction of the iceberg since they are used to their full potential. Animal-derived sequence-defined antibodies, including nanobodies (light blue circle), are cloned into recombinant formats. Consequently, these appear as a submerged fraction of the iceberg, having adopted all the undiscovered or underused versatility of recombinant formats. They also make up a small but expanding fraction in today's catalogs (below yellow line). However, the practicalities and limitations of using immunized sources restricts their range of uses. Similarly, non-animal-derived antibodies, in their various formats, applications and expression systems, also appear as submerged fractions below the waterline (below black line), being the most versatile since these consist of not only lgGs and other recombinant antibody formats (mostly Fab) but also new molecular entities like synthetic nanobodies and a growing portion of non-antibody scaffolds. Their common advantage is that they are the only antibody class that is always a sequence-defined reagent. Phage display using universal (that is, non-immunized) antibody libraries is by far the most used application for the new selection of recombinant binders. Consequently, it has demonstrated all the different application tweaks, such as affinity maturation, sandwich pair identification and cross reactivity competition ${ }^{16}$. Phage display antibodies also occupy a small fraction above the waterline since they are starting to appear in some catalogs, developed by dedicated companies and proudly announced as non-animal in origin (pink). In the center of the submerged fraction is a core of emerging new applications (green glow), radiating into all the applications, formats and expression systems of the submerged fraction. These applications require direct access to the gene of the antibody, as it will be cloned and expressed in new contexts.

overwhelmingly dominate the market. Such animal-derived molecules are generated using technologies developed over half a century ago. They continue to be widely used, despite the advantages offered antibodies meet the demands of the majority of uses and should be used as valid replacements for animal-derived antibodies in future antibody-generation experiments. In fact, the ability to unambiguously define antibodies by their sequences, when combined with stringent validation and quality control, provides substantially improved scientific reproducibility, as it can be assured that truly the same antibody is used, experiment after experiment ${ }^{6}$. To support higher scientific quality, regulatory standards and ethical core values, the limitations of animal-derived antibodies (described below) can be overcome via the use of universal antibody libraries.

Although such replacements would offer major scientific and societal benefits, non-animal-derived antibodies are not as widely available as animal-derived antibodies. So, as described in section 4 of the EURL ECVAM recommendation and to support a rapid transition to animal alternatives, the EU and its member states are encouraged to promote the generation and use of non-animal-derived antibodies. This should be achieved by education and training; pan-European, national and private funding efforts to improve accessibility; and the endorsement of replacement methods by government authorities, funding agencies and publishers. In this way, non-animal-derived antibodies will become as widely available as animal-derived antibodies are today. Ultimately, the hope is the use of antibody reagents uncharacterized at the molecular level will become as unacceptable as is the use of unsequenced plasmids or genes.

The research community has its part to play. Scientists should be incentivized to encourage this transition. If such commitments are made and upheld, researchers will be increasingly motivated to move away from animal-derived antibodies and to adopt non-animal-derived, better characterized antibodies.

\section{From the tip to below the surface}

Antibodies can be generated via several strategies (Fig. 2). The virtues of polyclonal antibodies derived from animal immunization are well known. They are cheap, do not demand advanced technical production skills and have particular advantages in applications where their heterogenous nature is beneficial for recognition of multiple epitopes. However, reliability is a major drawback. The vast majority of the antibodies present in an antiserum are directed against multiple targets, and unless the reagent is affinity purified, antibodies that react with the intended target are not enriched. 


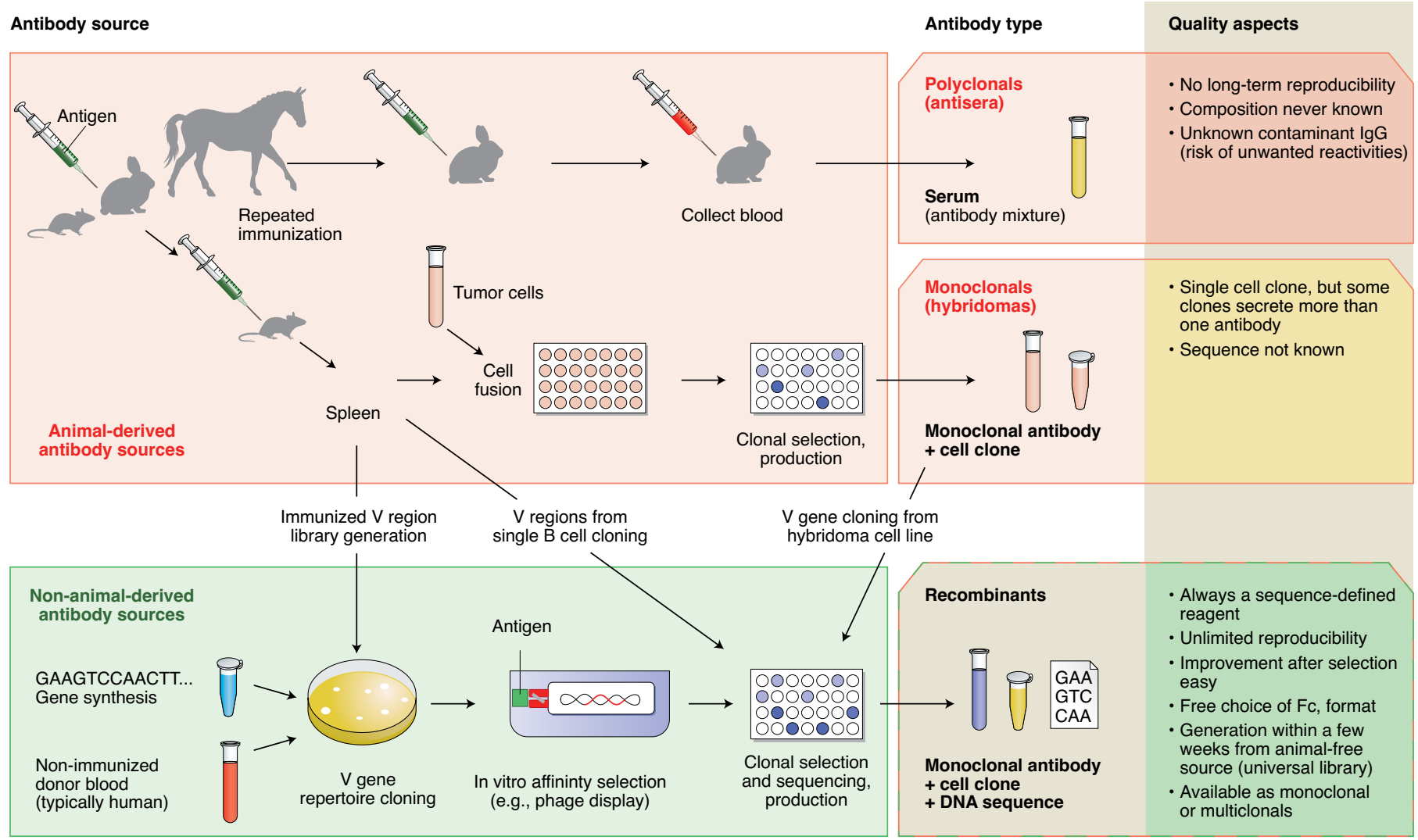

Fig. 2 | Antibody generation pathways. Immunization-dependent antibody generation methods were enabled with antisera from 1890 and hybridoma mAbs from 1975 onwards. The introduction of phage display in 1991 opened a path to using antibody gene libraries for enriching and isolating target-specific antibodies in vitro. The availability of new recombinant methods, when combined with strict quality control, created ways to improve on existing methods based on animal immunizations. Non-animal-derived methods typically deliver sequence-defined antibodies from the start, offering a more efficacious approach to reagent identification than is possible using immunization-based methods. Further, a standard phage display panning campaign requires less time than the minimum time needed by the immune system of any animal to produce an affinity-matured antibody, before both are subjected to fitness-for-purpose screening.

Recognition of multiple epitopes can also lead to unwanted cross-reactivities, high background and false-positive results ${ }^{8,9}$. Variability among different batches is unavoidable, even when they are produced in the same animal at different times. A particularly well-performing batch may be difficult to reproduce and is always a finite resource. This is a source of major frustration to end users.

mAbs derived from animal immunization do not suffer similar shortcomings. Derived from single cloned B cells, they have minimal batch-to-batch variability; the antibody generally binds with high specificity to only one epitope; and a continuous source of the isolated antibody is expected, as long as the clone can be revived from liquid nitrogen storage. However, the literature is littered with reports of non-specific mAbs ${ }^{10}$. Non-specificity can be an inherent property of an antibody, no matter how generated, but in the case of traditional monoclonals, there is an added problem: as an unavoidable consequence of the generation methods employed, many hybridomas express more than one antibody. A large multicenter study found extra, unexpected productive antibody genes in about a third of 185 randomly selected hybridomas, proving that a considerable fraction of hybridoma antibodies, despite being truly monoclonal, cannot be monospecific for genetic reasons inherent to their production ${ }^{5}$. Consequently, this calls into question all research that has relied on a particular non-monospecific clone $^{5}$ and mandates more care in the interpretation of results obtained with unsequenced hybridomas.

A last type of animal-derived $\mathrm{mAb}$ is also widely used; recombinant antibodies obtained by cloning antibody genes from hybridomas, single B-cell cloning, or generating antibody gene libraries from immunized animal B cells. Although these come with the advantages of recombinant antibodies, they require immunization and often generation of a new library every time.
In contrast to the above, non-animalderived recombinant antibodies are typically selected from high-diversity universal gene libraries generated by either cloning antibody gene repertoires from B cells from an unimmunized (naive) human donor, oligonucleotide synthesis guided by bioinformatic analysis of antibody sequences (Fig. 2), or combinations thereof. In vitro recombinant antibody generation methods rely on a variety of display systems, most commonly phage and yeast display. Although typically built using immunoglobulin domains, they are also complemented by an expanding literature on non-antibody-scaffold libraries.

\section{Advantages of in vitro-generated $\mathrm{mAbs}$} The ESAC mainly focused on phage display as an animal-free option because it is further advanced in terms of technological maturation and compatibility to most research applications. If phage display of universal libraries is used, animal 
immunization is not required at any point during antibody development and production. A large body of evidence supports the usefulness of phage display, with few perceived hurdles to its rapid implementation.

The ESAC report systematically reviews the performance of non-animal-derived antibodies, with examples spanning all the usual applications. The report highlights applications in western blotting, immunohistochemistry, immunocytochemistry, flow cytometry, enzyme-linked immunosorbent assays (ELISA), immunoprecipitation, immunosorbent assays and multiplexed planar or bead arrays ${ }^{11}$. There are many well-documented examples of applications where non-animal-derived antibodies and other affinity reagents have been generated and shown to perform at least as well as animal-derived antibodies.

In addition, several instances are identified where in vitro selection and the recombinant nature of an antibody has been proven to be a distinct advantage. For example, modification of panning conditions during phage display allows tuning of antibody target recognition properties; and sequence modification during cloning or synthesis can allow selection of suitable constant fragment $(\mathrm{Fc})$-free formats to solve flow cytometry issues, Fc-mutated formats to avoid non-specific binding by cellular Fc receptors, or incorporation of an affinity tag for analyte capture applications, such as proteomic microarrays ${ }^{12,13}$. One such microarray platform, composed of 349 non-animal-derived antibodies formatted as single-chain variable fragments (scFvs) directed against 156 antigens ${ }^{14}$, was successfully used to identify a biomarker signature associated with pancreatic stage I and II cancer.

As sequencing for initial identification of a clone is an integral part of the process for any recombinant antibody, non-animal-derived $\mathrm{mAbs}$ are unambiguously identifiable, guaranteeing a consistent, reproducible and unlimited supply (unlike polyclonal antibodies or mAbs produced from hybridomas). Although animal-derived recombinant $\mathrm{mAbs}$ share this advantage, a new immunized library must be produced for each new antigen of interest. In contrast, for non-animal-derived antibodies, the starting point is 'universal' or 'naive' libraries. The enormous diversity of candidate clones available in such libraries can yield antibodies against many different antigens, time and time again, equivalent to a lifetime supply of animals. For example, $\sim 10,000$ different $\mathrm{mAbs}$ have been generated from a single naive library in the Sanger Program ${ }^{15}$, and thousands of others have been generated as part of EU grant programs (for example, the EU Affinomics consortium, ID 241481).

The adaptability of non-animal-derived antibody libraries is already creating ripples of ingenuity and opening up applications beyond what can be achieved by relying on the natural immune system to generate diversity ${ }^{16}$. The ESAC report illustrates how huge libraries of structurally and genetically different candidate antibodies can be generated either by targeted mutation strategies or by creating synthetic diversity similar to, or greater than, that of the natural immune system. In vitro recombinant antibody technologies provide substantial advantages over immunization, including the ability to exceed the $\sim 100 \mathrm{pM}$ physiological limit of the $\mathrm{B}$ cell response and the ability to generate antibodies that recognize a broad range of toxic, non-immunogenic, pathogenic, conserved, or self targets. The unique capabilities of in vitro selection can improve characteristics such as stability, specificity and affinity. They also allow either counter-selection against structurally related competitors or the identification of cross-reactive antibodies with activity against analogs in different species, the profiles of which can be controlled during the experiment. Additionally, improved and more finely tuned candidates can be selected from sublibraries created by mutation strategies, followed by iterative cycles of panning using carefully controlled selection criteria.

These approaches, unique to universal display methods, open new vistas of specificity for target recognition. As the gene sequence is available, it is straightforward to apply the technology to changes in format, enzyme fusions, dimerization or multimerization, or addition of tags or fluorescent proteins. The versatility of these non-animal-derived antibodies means they can function as oligoclonals or intrabodies; they can also be expressed on the surface of a range of cells or viruses, with the latter allowing infection of particular cell types ${ }^{12,17-21}$. To retain the important multi-binding characteristics of polyclonals but without their limitations of poor definition and reproducibility, defined mixtures of non-animal-derived antibodies, called 'multiclonals', have been generated with low background, high specificity and unlimited reproducibility, and can now be used to replace animal-derived secondary antibodies ${ }^{22}$.

\section{Barriers to greater visibility and uptake}

Today, in a market flooded by animalderived antibodies, the most challenging aspect confronting the ESAC was to identify the major barriers to adoption and development of non-animal-derived antibodies and to understand the erroneous beliefs within the research community that have hindered their widespread use and contributed to their lack of visibility.

None of the negative perceptions has any basis in the presently achievable quality or molecular properties of non-animal-derived antibody reagents; in fact, in vitro recombinant antibody approaches are widely employed in the pharmaceutical industry for creating therapeutics, where products of the highest quality are required. Overall, the ESAC found several factors that contribute to the continued supply and use of animal-derived antibodies in the basic research market, despite the availability of an alternative method of generation: first, there is inertia and a propensity to use existing or familiar methods; second, in most fields of biological research, there is ignorance of the advanced level now attained by non-animal-derived antibody technology; third, there are perceived economic and legal constraints for companies looking to enter the reagent market for in vitro antibody technology; and finally, access to non-animal-derived antibody resources, either from commercial sources or from academic centers, is limited.

The widespread acceptance of mediocre antibody quality by many biologists ${ }^{23}$ is compounded by the continued commercial supply of animal-derived products and a shortage of companies providing non-animal-derived antibody generation services. The inherent shortcomings of many animal-derived antibodies in supplier catalogs are accepted, while early negative experiences with recombinant prototypes are incorrectly perceived as reasons for basic researchers to avoid non-animal-based alternatives. Despite the abundance of validating publications, a lack of understanding in the use of recombinant methods persists.

A focus by in vitro recombinant antibody technology developers on the more lucrative therapeutics market and intellectual property restrictions surrounding these methods has also inhibited widespread commercial diffusion of the technology. This situation is changing, however. The ESAC report appendix, section III, details the availability of non-animal-derived custom antibody production services and reagents, highlighting a growing number of suppliers (see also http://www.afability.com for updates) and academic consortia that have successfully generated thousands of binding reagents. 
Certainly, the establishment of molecular display techniques in laboratories unfamiliar with them can be challenging. Naive recombinant library development (from natural or synthetic sources) requires skilled molecular biology expertise and a substantial initial time investment. However, the diversity of acquired antibody candidates, the longevity of libraries after initial construction and the abolition of ongoing costs of animal care make this effort worthwhile. Once a library is available, the cost of generating and validating non-animal-derived antibodies is in the same range as the cost of animal-derived mAbs, requiring standard molecular biology laboratory equipment and consumables.

As demand increases, high-throughput technologies already solidly established in the therapeutic antibody generation market are expected to be adopted in the life sciences market and further price reductions are expected. Beside cost, there is a major benefit in time as antibodies can be selected from a naive recombinant library in a matter of a few weeks, as impressively demonstrated by the generation of recombinant SARS-CoV-2 binders in as little as four weeks by a host of academic and industrial groups (for example, Antibody Therapy Against Coronavirus, Yumab/Boehringer Ingelheim, CORAT Therapeutics, Molecular Partners, Avant Gen, Chugai Pharmabody Research/Agency for Science, Technology and Research, Centivax/Distributed Bio, Specifica and AstraZeneca; see https://www. antibodysociety.org/covid-19/).

Another commonly expressed misconception is that, to exploit the full potential of the immune repertoire, a whole-animal immune response to an antigen is required. This misconception has been contradicted by the many successfully applied efforts to generate antibodies from universal libraries to a large number of antigens, including SARS-CoV-2. The ESAC report describes how the non-animal-derived antibody development strategy has adopted and adapted the essential principles of the naturally evolving immune system during the generation of antibody candidates against a specific target. Specifically, the underlying mechanistic, functional, structural and diversity-generating similarities are clarified.

Scientific misconceptions surrounding sensitivity and avidity have also influenced a reluctance to adapt to certain formats, such as a monovalent antibody lacking an Fc region. Yet the submerged fraction of the antibody iceberg bestows on us the opportunity to work with a variety of alternative formats, with parameters that render them functionally indistinguishable from animal-derived antibodies in all typical applications. Today, recombinant production of the full immunoglobulin G (IgG) is straightforward, and in certain applications the use of the antibody fragment enjoys its own distinct advantages. Despite decades of development and maturity of a growing list of expression systems, formats, detection methods, picomolar affinities and application to every possible assay, a perception bias against display methods of antibody generation persists in parts of the research community, possibly a holdover from early publications from the 1990s, when the technologies were first introduced and were not as advanced as they are today. In sharp contrast, although not prevailing exclusively, the use of animal-free antibody generation methods by the pharmaceutical industry is now well established, and the clinical success of animal-free $\mathrm{mAb}$ products is the ultimate validation of antibody quality ${ }^{24}$.

Given that any antibody - whether generated by immunization or in vitro display methods - may lack intrinsic specificity for a target; may cross-react as a result of fortuitous interactions with the antibody binding site (lack of specificity); may bind to related epitopes occurring in different antigens, such as homologous proteins (lack of selectivity); or may preferentially bind to folded (conformational) or unfolded (linear) epitopes, the ESAC report stresses the importance of subjecting any affinity reagent to stringent quality control and validation. Sequencing of an antibody, regardless of its source, is important to assure the identity of the reagent and improve reproducibility. The very strict quality control imposed on therapeutic antibodies by the US Food and Drug Administration or European Medicines Agency assures that these goals are met.

The antibody supplier market is, however, a different matter. The inadequate validation of antibodies used in research settings results in a lack of antibody specificity that plagues scientific reproducibility, with a pernicious effect on science in terms of wasted money, opportunity, time, and human or other resources ${ }^{6}$.

An opportunity exists for antibody manufacturers operating under EU regulation and beyond to develop antibodies by using non-animal-derived antibody technology and for end users to adopt them. The scientific community should also demand a better quality of antibodies from commercial sources worldwide. If improved quality is demanded, manufacturers of existing animal-derived antibodies will have to do the extra time- and cost-consuming sequencing analysis and validation to measure their specificity and performance. As sequencing is routinely part of the process for generating non-animal-derived antibodies, and in conjunction with other advantages such as speed of generation, greater range of targets and opportunities to take advantage of challenging selection conditions, this will give companies using these technologies a clear economic advantage. In return, the scientific community stands to gain improved reproducibility, a readily available and renewable source of antibody reagents, and a reagent with greater versatility. Thus, the adoption of non-animal-derived recombinant antibody technology over immunization-based methods represents a scientific, ethical and societal imperative.

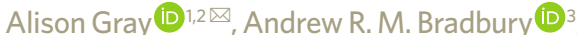 Achim Knappik (D) ${ }^{4}$, Andreas Plückthun (D) Carl A. K. Borrebaeck (D) ${ }^{6}$ and Stefan Dübel(D) ${ }^{1}$ Afability, Nottingham, UK. ${ }^{2}$ School of Veterinary Medicine and Science, University of Nottingham, Loughborough, UK. ${ }^{3}$ Specifica, Santa Fe, NM, USA. ${ }^{4}$ Bio-Rad, Puchheim, Germany. ${ }^{5}$ University of Zurich, Zurich, Switzerland. ${ }^{6}$ University of Lund, Lund, Sweden. ${ }^{7}$ Technische Universität Braunschweig, Braunschweig, Germany.

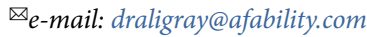

Published online: 12 October 2020

https://doi.org/10.1038/s41587-020-0687-9

References

1. Gray, A. C., Sidhu, S. S., Chandrasekera, P. C., Hendriksen, C. F. M. \& Borrebaeck, C. A. K. Trends Biotechnol. 34, 960-969 (2016).

2. Gray, A. C., Sidhu, S. S., Chandrasekera, P. C., Hendriksen, C. F. M. \& Borrebaeck, C. A. K. Science 353, 452-453 (2016).

3. Directive $2010 / 63 / \mathrm{EU}$ of the European Parliament and of the Council of 22 September 2010 on the protection of animals used for scientific purposes. http://data.europa.eu/eli/dir/ 2010/63/oj (2010).

4. Barroso, J., Halder, M. \& Whelan, M. EURL ECVAM recommendation on non-animal-derived antibodies. EUR 30185 EN (European Union, 2020); https://doi.org/10.2760/091625

5. Bradbury, A. R. M. et al. MAbs 10, 539-546 (2018).

6. Bradbury, A. \& Plückthun, A. Nature 518, 27-29 (2015).

7. Baker, M. Nature 521, 274-276 (2015).

8. Michel, M. C., Wieland, T. \& Tsujimoto, G. Naunyn Schmiedebergs Arch. Pharmacol. 379, 385-388 (2009).

9. Russo, G. et al. N. Biotechnol. 45, 98-112 (2018).

10. Kramer, A. et al. Cell 91, 799-809 (1997).

11. Uhlen, M. et al. Nat. Methods 13, 823-827 (2016).

12. Frenzel, A. et al. Transfus. Med. Hemother. 44, 312-318 (2017).

13. Steinhauer, C. et al. Proteomics 6, 4227-4234 (2006).

14. Mellby, L. D. et al. J. Clin. Oncol. 36, 2887-2894 (2018)

15. Schofield, D. et al. Genome Biol. 8, R254 (2007).

16. Bradbury, A. R., Sidhu, S., Dübel, S. \& McCafferty, J. Nat. Biotechnol. 29, 245-254 (2011)

17. Batista, F. D. \& Neuberger, M. S. Immunity 8, 751-759 (1998).

18. Zahnd, C., Sarkar, C. A. \& Plückthun, A. Protein Eng. Des. Sel. 23, 175-184 (2010)

19. Luginbühl, B. et al. J. Mol. Biol. 363, 75-97 (2006).

20. Geyer, C. R., McCafferty, J., Dübel, S., Bradbury, A. R. \& Sidhu, S. S. Methods Mol. Biol. 901, 11-32 (2012).

21. Buchholz, C. J., Friedel, T. \& Büning, H. Trends Biotechnol. 33, $777-790$ (2015)

22. Wenzel, E. V., Russo, G. \& Dübel, S. BIOspektrum (Heidelb.) 26, 416-417 (2020) 
23. Goodman, S. L. N. Biotechnol. 45, 9-13 (2018).

24. Frenzel, A., Schirrmann, T. \& Hust, M. MAbs 8, 1177-1194 (2016).

Competing interests

A.R.M.B.: CSO, cofounder and shareholder of Specifica Inc., involved in the supply of antibody library and discovery services for the therapeutic market. S.D.: shareholder of companies that employ animal-free antibody generation - namely, mAb-factory $\mathrm{GmbH}$,
Yumab GmbH and Abcalis GmbH. A.G.: founder of AFABILITY, a non-profit organization that advises on antibody production methods and scientific procedures involving the use of animals according to Directive 2010/63/EU. A.P.: cofounder and shareholder of companies (Morphosys AG, Molecular Partners AG) that employ animal-free antibody and binding protein generation for therapeutics. A.K.: shareholder of companies (Morphosys AG, Molecular Partners AG, Bio-Rad Laboratories) that employ animal-free antibody and binding protein generation. All authors are independent advisors on the EU ECVAM scientific advisory committee (ESAC) for the scientific validity of alternative methods for antibody production. ESAC is composed of external scientists who are appointed on the basis of their scientific expertise; they act independently, in the public interest, and do not represent their company's, the EU's or external interests of any kind.

\section{A novel computational architecture for large-scale genomics}

To the Editor - Biomedical and biological research is increasingly shifting toward the collection and analysis of ever-larger datasets. For example, genomics for precision medicine is moving from bulk sequencing toward single-cell studies that substantially increase the amount of data collected $^{1,2}$; large-scale human research, clinical trials and population studies are also generating ever larger datasets ${ }^{1,3-5}$; and biological and biomedical fields such as microscopy, medical imaging or wearable devices are also increasingly generating gigabytes to terabytes of data.

At the same time as the datasets are increasing in size, our ability to develop more powerful processors for data analysis is becoming limited by our ability to further miniaturize silicon transistors ${ }^{6}$; as a result, alternative computational approaches are needed. Here we introduce memory-driven computing (MDC) as a novel and alternative in silico architecture that overcomes many of the limitations posed by current approaches.

The increasing data avalanche poses multiple challenges for our computing resources: first, processing time needs to be maintained in the presence of an exponential increase in data volume; second, costs from energy consumption need to be managed while providing more computational capacity; third, data movement and duplication need to be minimized across multiple locations (for example, at data collection sites and the institutions analyzing data); fourth, data and privacy protection need to be managed on the basis of regional regulations (for example, the US Health Insurance Portability and Accountability Act of 1996 (HIPAA) or the European Union's General Data Protection Regulation 2016/679; GDPR) ${ }^{7}$; and fifth, clinical applications require substantially faster processing

a

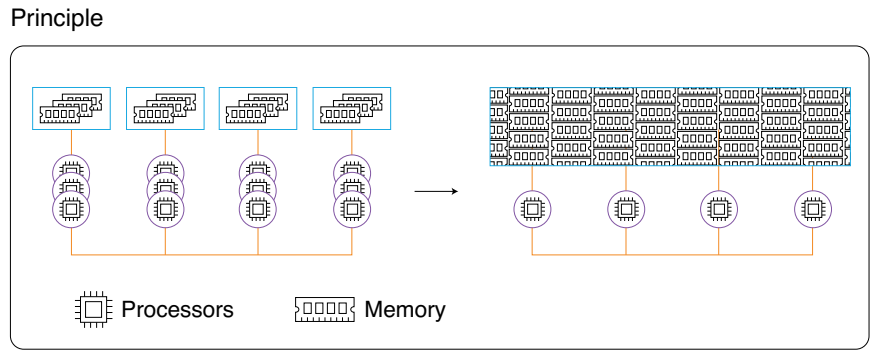

b

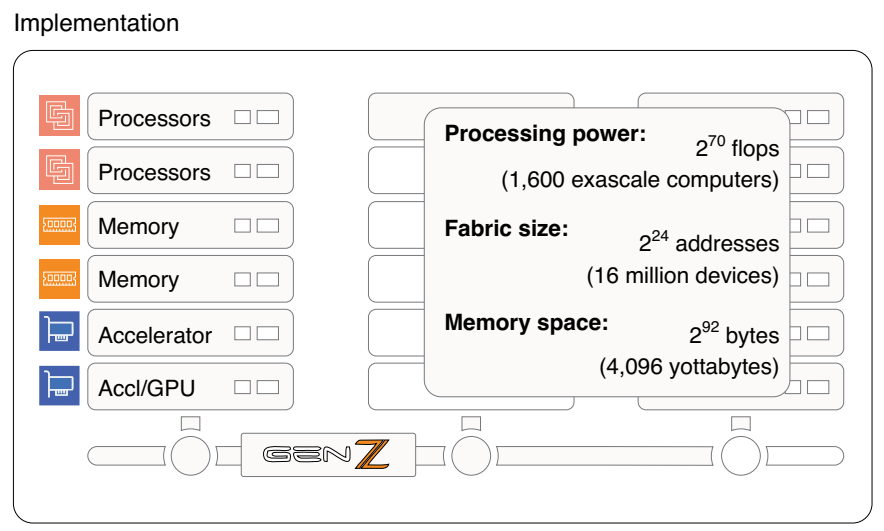

Fig. 1 | Principles and implementation of an MDC architecture. a, Principles of traditional cluster architecture (left) and MDC architecture (right). b, Implementation of MDC with large, shared and persistent memory, which connects this memory and processors, accelerators (Accl) and graphics processing units (GPUs) via a fabric following the Gen-Z protocol.

times - for example, when preprocessing raw data for analysis.

MDC is a novel computer architecture that is specifically tailored toward data-driven research. Traditional scale-out computational clusters with many computing nodes can parallelize computation but require data to be partitioned across the computing cluster. Because data need to be shared across processing units, they are passed across so-called I/O (input/output) networks using messaging (Fig. 1a, left), which limits performance at scale unless the algorithm is excessively parallel. Alternatively, data can be held in one place in a scale-up architecture with larger memory; however, such scale-up machines are often limited by the number of processors that can be managed in the computing architecture.

By building on the benefits of the above two traditional architectures without their drawbacks, MDC has the potential to complement traditional networks through a 